\title{
VII.-The Effect of Temperature and of Pressure on the Limits of Inflammability of Mixtures of Methane and Air.
}

By Walter Mason and Richard Vernon Wheeler.

A "LIMIT" mixture of an inflammable gas with air or oxygen can be defined as such that the heat evolved by the combustion of one "layer" of the mixture is sufficient, and only just sufficient, to raise to its ignition-temperature the layer adjacent, so that flame once started in such a mixture continues to be propagated progressively throughout, without the necessity for the continued presence of the source of heat that caused the inflammation.

The self-propagation of flame through the mixture is only possible when the speed of reaction between tha combining gases is sufficient to overcome any loss of heat, by radiation, convection, and conduction, under the conditions of its combustion. The speed of reaction is dependent on the temperature that the portion of the mixture which is burning can impart to the portion that is about to burn.

It follows naturally, therefore, that the higher the initial temperature of the mixture the less is the propagation of flame dependent upon the heat generated by combustion, so that the effect of increasing the initial temperature of mixtures of methane and air should be to widen their limits of inflammability, lowering the lower and raising the upper limit.

The effect of pressure on the limits is not so easy to forecast; in point of fact, the results of experiments did not fulfil our anticipations. It will be best to leave discussion of the matter until the experiments have been described.

\section{The Effect of Temperature.}

The earliest systematic experiments on the effect of temperature on the limits of inflammability of gaseous mixtures seem to have been by Bunte and Roszkowski in 1890 (J. Gasbeleuchtung, 1890, 33, 491, 524, 535, 553). The mixtures were ignited electrically in a spherical vessel of 35 c.c. capacity. Apart from the fact that it is impossible to judge accurately as to the propagation of flame in such a vessel, something must have been radically wrong with Bunte and Roszkowski's experimental arrangements, for they recorded no marked change in the limits for mixtures of methane 
and air when the initial temperature of the mixtures was raised from $15^{\circ}$ to $300^{\circ}$.

Taffanel (Compt. rend., 1913, 157, 593) examined the effect of increased temperature on the lower limit for mixtures of methane and air by passing an electric spark in the mixtures, after introducing them into a tube at the required temperature, and observing whether or no inflammation was propagated throughout the mixture. His results were as follow:

\begin{tabular}{|c|c|c|c|c|c|c|}
\hline ture ............................... & $20^{\circ}$ & $175^{\circ}$ & $237^{\circ}$ & $312^{\circ}$ & $555^{\circ}$ & $690^{\circ}$ \\
\hline$\ldots \ldots, \ldots, \ldots$ & $5 \cdot 80$ & $5 \cdot 25$ & $4 \cdot 75$ & $4 \cdot 30$ & $3 \cdot 40$ & $3 \cdot 00$ \\
\hline
\end{tabular}

Taffanel did not state the dimensions of his tube, the position of the point of ignition of the mixtures, or the direction of travel of the flames; but from his results, which, as will be seen later, closely correspond with our own, we conclude that a rather wide and short tube was used and that ignition was at the top, the flames being propagated vertically downwards.

Burrell and Robertson (United States Bureau of Mines, Technical Paper No. 121, 1916) repeated Taffanel's experiments, using a Hempel bulb of 100 c.c. capacity as the explosion vessel and igniting the mixtures near the top by an electric spark. The bulb was heated in an electric furnace, and whether or no flame had propagated throughout the mixture was judged by analysing the products of combustion. The results recorded were:

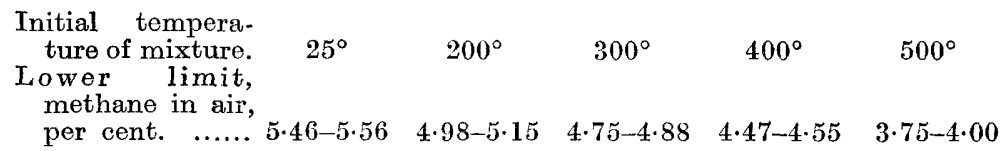

Accurate observation of the propagation of flame in a vessel that is totally enclosed in an electric furnace presents some difficulty. It is not easy to judge, for example, of the extent of downward propagation of flame in a tube by an arrangement of mirrors, since the flame is viewed end-on; and analysis of the gases remaining after flame has passed, not altogether satisfactory as a means of judging whether flame has travelled or has just failed to travel throughout the vessel with lower-limit mixtures, gives quite misleading results with the upper-limit mixtures where much of the combustible gas remains unburnt or incompletely burnt.

For our experiments we have made use of the fact that the limits for upward propagation of flame are wider than for downward propagation (T., 1914, 105, 2591) to aid in observing whether 
or no flame travelled throughout the mixtures at different temperatures, in the manner described in the experimental portion of this paper. The criterion of inflammability adopted was self-propagation of flame downwards. The gaseous mixtures were stored over

Fig. 1.

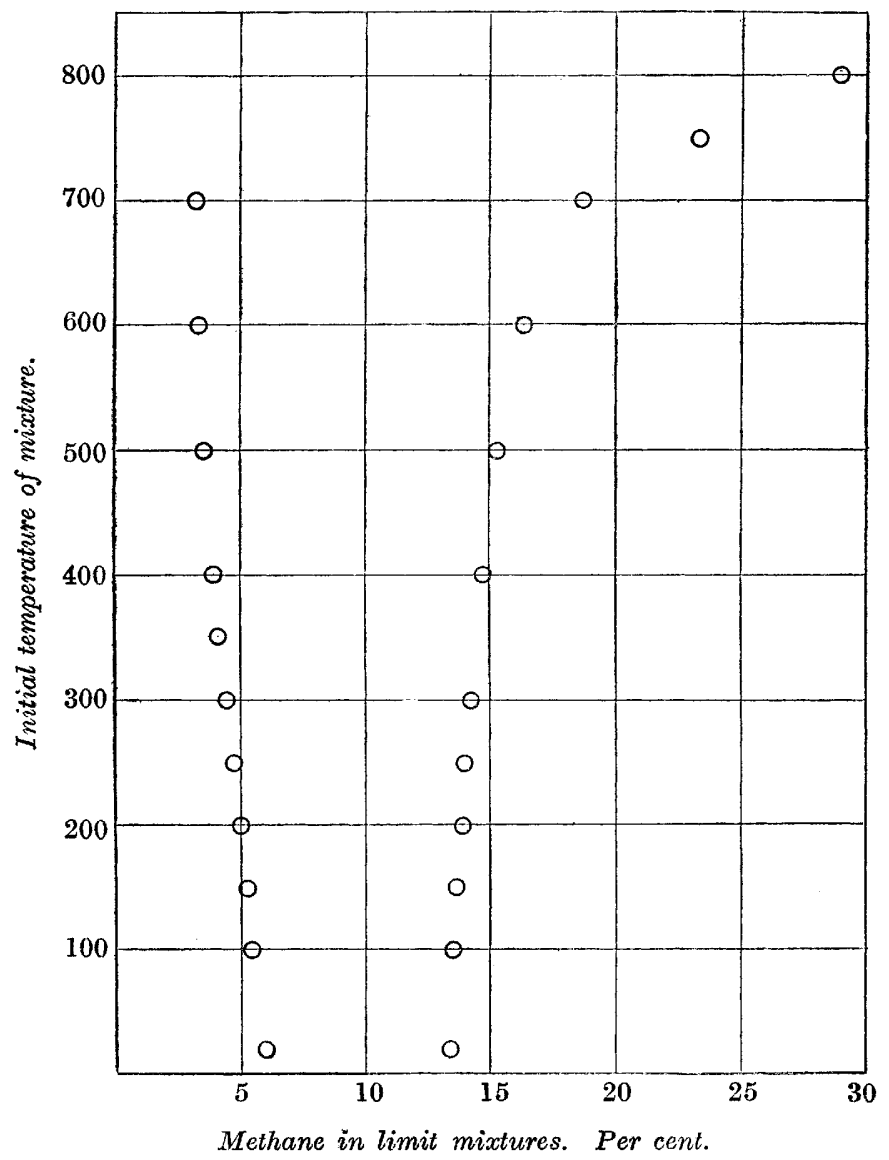

a mixture of equal parts by volume of glycerol and water in glass gas-holders, whence they were introduced into the previously heated and evacuated explosion vessel; they were thus saturated with water vapour at the room temperature $\left(20^{\circ}\right)$. The results are given in the table that follows and diagrammatically in Fig. 1. 
Downward propagation of flame in mixtures of methane and air. Methane, per cent.

Initial temperature.

$20^{\circ}$
100
150
200
250
300
350
400
500
600
700
750
800

\begin{tabular}{|c|c|}
\hline Lower limit. & Upper limit. \\
\hline 6.00 & $13 \cdot 40$ \\
\hline $5 \cdot 45$ & $13 \cdot 50$ \\
\hline $5 \cdot 20$ & $13 \cdot 60$ \\
\hline $5 \cdot 05$ & $13 \cdot 85$ \\
\hline $4 \cdot 60$ & $14 \cdot 00$ \\
\hline $4 \cdot 40$ & $14 \cdot 25$ \\
\hline $4 \cdot 15$ & not determined. \\
\hline $4 \cdot 00$ & $14 \cdot 70$ \\
\hline $3 \cdot 65$ & $15 \cdot 35$ \\
\hline $3 \cdot 35$ & $16 \cdot 40$ \\
\hline $3 \cdot 25$ & 18.75 \\
\hline - & $23 \cdot 60$ \\
\hline — & $29 \cdot 00$ \\
\hline
\end{tabular}

The mixtures were allowed to remain during two seconds in the explosion vessel (which had been previously brought to the required temperature) before the passage of an electric spark to cause ignition. This length of time was sufficient to ensure that the gases attained the temperature of the enclosure (compare Burrell and Robertson, loc. cit., p. 6), and at the same time, except perhaps at the highest temperatures, did not admit of appreciable combustion on the surface of the vessel before the spark was passed.

Consider first the lower limit. Progressive increase of the initial temperature causes a corresponding decrease in the amount of methane required in the air to enable self-propagation of flame to take place. Our determinations agree closely with Taffanel's, and therefore disprove Bunte and Roszkowski's results. With an initial temperature of $750^{\circ}$, a mixture containing 3.00 per cent. of methane ignited without being sparked (and flame was propagated throughout) as soon as it entered the explosion vessel, the rate of reaction on the surface of the explosion vessel at this temperature being sufficiently rapid to cause self-heating of the mixture. At $700^{\circ}$ no ignition occurred with a 3.20 per cent. mixture by the walls of the vessel, nor was flame propagated throughout such a mixture when an electric spark was passed; for complete propagation of flame at this temperature the mixture had to contain 3.25 per cent. of methane. Under the conditions of these experiments, therefore, the ignition-temperature (that is, the temperature at which rapid self-heating takes place) of a 3 per cent. methane-air mixture can be regarded as between $700^{\circ}$ and $750^{\circ}$ (compare Taffanel, loc. cit., p. 597).

Just as the lower limit is lowered by increasing the initial temperature, the upper limit is raised. The extent to which the limit is raised increases regularly with the initial temperature of 
the mixture until $600^{\circ}$ is exceeded, after which there is a considerable rise in the rate of increase (see Fig. 1). This is probably due to reaction between methane and oxygen on the surface of the explosion vessel being sufficiently rapid with high concentrations of methane at temperatures above $600^{\circ}$ to alter the constitution of the mixture during the two seconds that elapse between its introduction into the heated explosion vessel and the passing of the spark. We know that with excess of methane in air, carbon monoxide and hydrogen (both of which have very high upper limits of inflammability) persist in the products of combustion, and it may be that the early production of these gases is the cause of the abnormal raising of the upper limit of inflammability of mixtures of methane and air at temperatures greater than $600^{\circ}$. That combustion without flame takes place to an appreciable extent above $600^{\circ}$ under the conditions of the experiments is shown by the fact that at $700^{\circ}$ no ignition could be obtained (with a mixture containing 18.70 per cent. of methane) if the mixture were left in the explosion vessel longer than five seconds before sparking, whilst at $750^{\circ}$ an interval of only 1.5 seconds sufficed to render a mixture containing 23.50 per cent. of methane uninflammable. In each of these instances we can assume that the mixtures became uninflammable by deprivation of oxygen consequent on its burning the methane. If, therefore, the formation of carbon monoxide and hydrogen by combustion of methane is the cause of the abnormal raising of the upper limit at these high temperatures, the effect is transitory. Hydrogen would be produced also by thermal decomposition of methane, but the reaction is very slow at temperatures below $900^{\circ}$. In any event, it is clear that the results obtained at temperatures above $600^{\circ}$ do not properly represent the upper limits for self-propagation of flame in mixtures of methane and air.

It may be mentioned that in no instance did ignition of, and propagation of flame in, a high-limit mixture take place through contact with the heated walls of the explosion vessel. At $800^{\circ}$, for example, a spark had to be passed in order to cause flame to travel through the mixture (containing 28.70 per cent. of methane).

\section{The Effect of Pressure.}

The effect of initial pressure above atmospheric on the limits of inflammability of mixtures of hydrogen, carbon monoxide, and methane, respectively, with air has been studied by Terres and Plenz (J. Gasbeleuchtung, 1914, 57, 990, 1001, 1016, 1025). The explosion vessel used was an iron cylinder $37 \mathrm{~cm}$. long and $8 \mathrm{~cm}$. in diameter. Ignition was by an electric spark at a point $4 \mathrm{~cm}$. 
from the top of the cylinder and on its vertical axis. The criterion of inflammability was, therefore, self-propagatio 1 of flame downwards. Whether or no flame had passed throughout the mixture was judged by analysing the products of combustion.

For methane (firedamp from Kissarmas, Transylvania, containing 99.1 per cent. of methane), Terres and Plenz obtained the results shown in the tables that follow.

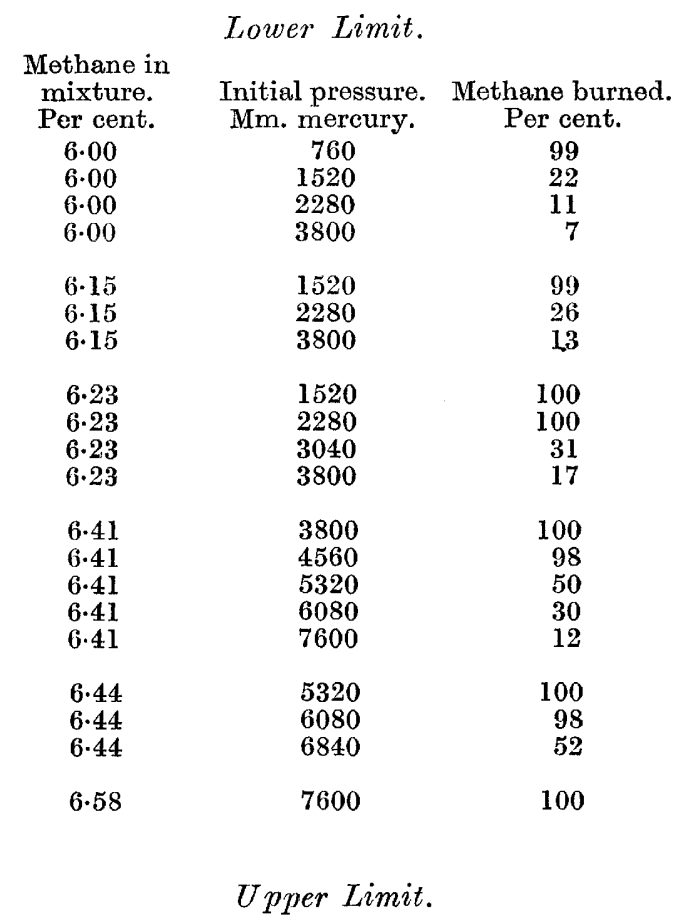

\section{Mothane in mixture. Per cent.} $12 \cdot 98$

$13 \cdot 35$

$13 \cdot 65$

$13 \cdot 98$
Initial pressure required for propagation of flame. Mm. mercury.

$$
\begin{array}{r}
760 \\
2280 \\
4560 \\
6840
\end{array}
$$

With upper-limit mixtures, according to Terres and Plenz, either the mixture did not burn at all under the conditions of their experiments or flame travelled throughout. No partial propagation of flame took place as with the lower-limit mixtures. Their experiments can be summarised in the statement that an increase of the initial pressure of the mixtures to 10 atmospheres 
raised the lower limit (downward propagation of flame) from 6.0 to 6.5 per cent., and also raised the upper limit from 13.0 to 14.0 per cent.

Burrell and Robertson (loc. cit.) also studied the effect of pressure on the limits of inflammability of mixtures of methane and air, using the same apparatus as for their work on the effect of temperature. Their experiments were mainly at pressures less than atmospheric, but they recorded that "increasing the initial pressure up to 5 atmospheres had no effect in changing the low limit of complete propagation" (p. 10). At reduced pressures, they found that the limits were narrowed, until at pressures between 250 and $300 \mathrm{~mm}$. of mercury it was impossible, under the conditions of their experiments, to obtain self-propagation of flame in any mixture of methane and air.

Our experiments at reduced pressures have been made with initial temperatures of $20^{\circ}, 250^{\circ}$, and $500^{\circ}$. The same apparatus was used as for the experiments with different initial temperatures at atmospheric pressure. The results are shown diagrammatically in Fig. 2.

The fact that Burrell and Robertson were unable to obtain selfpropagation of flame in any mixture at pressures less than $300 \mathrm{~mm}$. (at atmospheric temperature), whereas we found the limiting pressure to be $120 \mathrm{~mm}$., is explicable on the assumption that their means of ignition was inadequate. For example, a mixture of methane and air containing 9.5 per cent. of methane can be ignited at atmospheric pressure by the secondary discharge from an "8-inch" $X$-ray coil at a spark-gap of $1 \mathrm{~mm}$. between platinum points when the current broken in the primary circuit (the trembler being locked) is about 0.5 ampere; but when the pressure of the mixture is reduced to $100 \mathrm{~mm}$., it is necessary to break a current of more than 7 amperes in the primary circuit of the same coil in order to obtain a secondary discharge at a $1 \mathrm{~mm}$. gap capable of igniting it (T., 1917, 111, 130). Burrell and Robertson's results express, not the limiting pressure for self-propagation of flame in any mixture of methane and air, but the limiting pressure for ignition by a secondary discharge of the particular intensity employed by them.

Examination of Fig. 2 shows that at atmospheric temperature the mixtures of methane and air in which flame is propagated (downwards) most readily, as evinced by their having the lowest limiting pressure, contain between 8.75 and 9.40 per cent. of methane.

The same conclusion can be drawn from the experiments by Burgess and Wheeler on "limit" mixtures of methane, oxygen, 
and nitrogen (T., 1914, 105, 2596). Those experiments showed that by the gradual substitution of nitrogen for the oxygen in air the limits were narrowed until, when the "atmosphere" contained only 13.45 per cent. of oxygen, they lay between 6.50 per cent. (lower) and 6.70 per cent. (upper) of methane. From the partial pressures of the gases, the percentages of methane in the atmospheric air contained in these limit mixtures can be calculated, the presence of the excess of nitrogen and of methane (above that

Frg. 2.

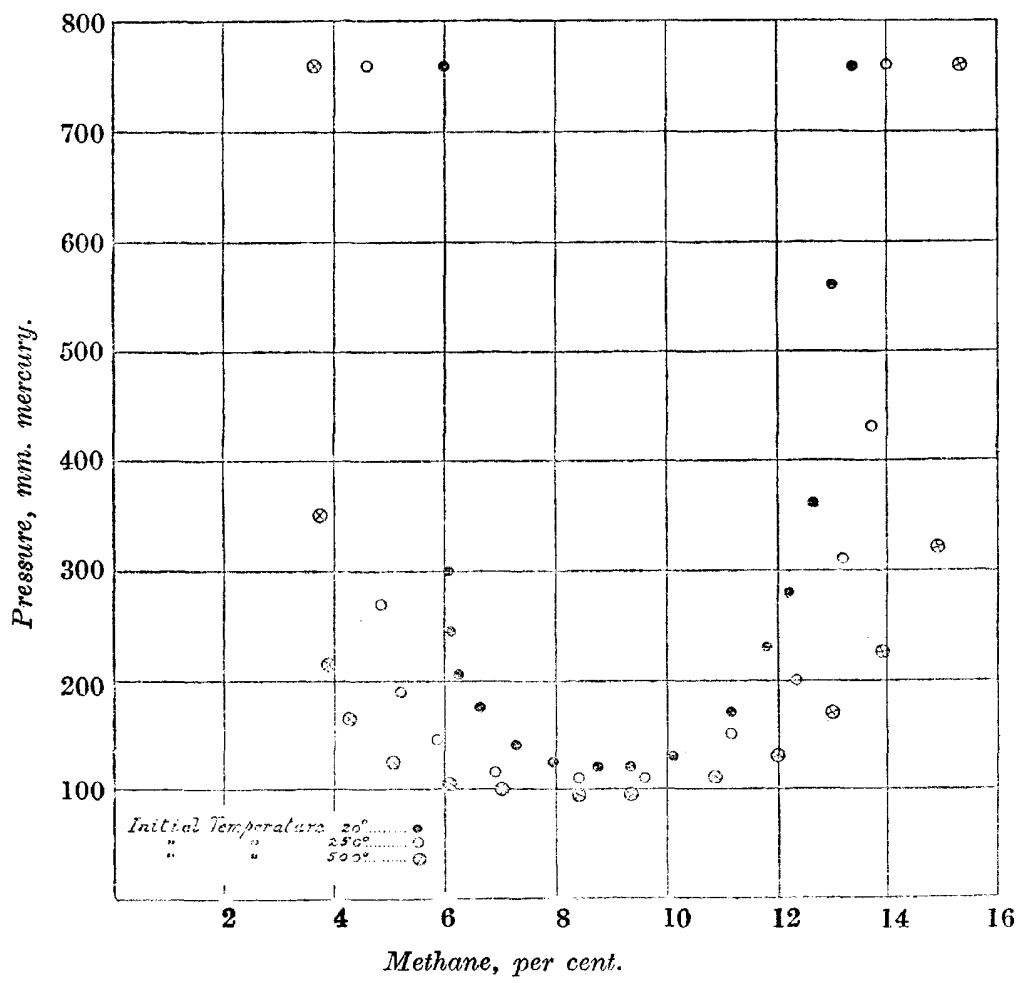

required for complete combustion by the oxygen) being regarded as equivalent to a reduction of pressure. This calculation gives 9.2 per cent. of methane in air as the mixture that propagates flame most readily.

The mixtures of methane and air most readily ignited by a secondary discharge, or by the break-flash ("momentary arc") produced at the point of fracture of a metallic electric circuit (continuous current), contain between 8.0 and 8.6 per cent. of 
methane, whilst the mixtures in which the "uniform movement" of propagation of flame is most rapid contain between 9.5 and 10.0 per cent. of methane (T., 1914, 105, 2607).

The range of mixtures over which propagation of flame occurs most readily thus lies mid-way between the range over which ignition (by electrical means) is easiest and the range over which the flames are fastest. It might be argued from this that ease of propagation of flame in a series of mixtures of methane and air is dependent about equally on the ease of ignition of the mixtures and on the ratio $T-t / t-\theta$ (see T., 1917, 111, 1044). It must be remembered, however, that the ignitibility of the mixtures, as shown by the intensity of the secondary discharge or the primary break-flash required to ignite them, is not necessarily a measure of their ignitibility by heated gases during the propagation of flame.

The effect of raising the initial temperature of the mixtures is to widen the dilution limits at all pressures, and the limiting pressure for the self-propagation of flame in any mixture is lowered, though not to a great extent. A point in these experiments that should be noted is the small effect on the limits produced by reducing the initial pressures by as much as half an atmosphere; the effect does not begin to be marked until the pressure is reduced below $300 \mathrm{~mm}$.

For experiments with initial pressures greater than atmospheric, we have used a tube of stout glass into which the previously prepared mixtures could be forced through a condensing syringe in the manner described in the experimental portion of this paper. The criterion of inflammability was, as before, propagation of flame downwards. The results were as follow:

Initial pressure. Mm. mercury. 760
1250
2100
2900
3350
3750
4650
Lower limit. Methane, per cent. 6.00 6.05 not determined. $6 \cdot 20$ $6 \cdot 25$

not determined. $6 \cdot 40$
Upper limit.

Methane, per cent.

$13 \cdot 00$

$13 \cdot 15$

$13 \cdot 35$

$13 \cdot 60$

not determined.

$13 \cdot 80$

$14 \cdot 05$

We thus confirm Terres and Plenz's results. For the lowerlimit mixtures, our values agree closely with theirs, but we obtained a greater raising of the upper limit by increasing the pressure than they recorded. This, we think, is due to Terres and Plenz not having used a sufficiently powerful source of ignition, for they have stated that the mixtures either did not ignite at all or flame travelled throughout them. This is contrary to our ex- 
perience, for we obtained many mixtures at high pressures in which flame travelled only part of the way down the tube. Mixtures of methane and air containing more than 11 per cent. of methane require a secondary discharge of considerable intensity to ignite them, and the intensity required increases rapidly with increased methane content. Thus, a mixture of methane and air, at atmospheric pressure, containing 12 per cent. of methane, can be ignited by the secondary discharge from an "8-inch" $X$-ray coil across a spark-gap of $3 \mathrm{~mm}$. when a current of 7.5 amperes is broken in the primary circuit (the trembler being locked), whereas a mixture containing 13 per cent. of methane requires 15 amperes, and one that contains 14 per cent. 32 amperes. On the other hand, an increase of the pressure (above atmospheric pressure) of any of the mixtures renders it capable of being ignited by a discharge of slightly less intensity (T., 1917, 111, 411).

It is easy to see, therefore, by the manner in which they expressed their results, how Terres and Plenz confused the inability of their secondary discharge to ignite some of their mixtures with incapability of the mixtures to propagate flame They recorded, for example, that a mixture containing 13.65 per cent. of methane did not propagate flame-did not burn at all-when the initial pressure was 6 atmospheres; on raising the pressure to 7 atmospheres (when the mixture would be rendered more readily ignitible), flame was propagated throughout.

Leaving differences in degree aside, however, we are in agreement with Terres and Plenz in observing a raising of both the lower and the upper limit with increased initial pressure (above atmospheric), and we find the results difficult to explain. From the law of mass action, we anticipated that increased pressure, if it had any appreciable effect after atmospheric pressure had been passed, would widen the limits on both sides. If the widening of the upper limit be explained by mass action, what is the reason for the narrowing of the lower limit?

According to the kinetic theory, loss of heat from gases by conduction and radiation is independent of the pressure. When attempting to put this deduction from the kinetic theory to experimental proof, however, Kundt and Warburg (J. de Physique, $1876,5,118)$ found that unless the pressure of the gas was low, the loss of heat due to conduction was masked by that arising from convection currents. It may be, therefore, as Terres and Plenz have suggested, that the loss of heat from a gas at high pressure is due to a greater extent to convection than to conduction, and increases with the pressure. According to this explanation, the upper limit should also be narrowed, and when hydrogen 
or carbon monoxide is the combustible gas this is so. What must be regarded as the abnormal behaviour of mixtures of methane and air under pressure at the upper limit requires further study.

Frg. 3.

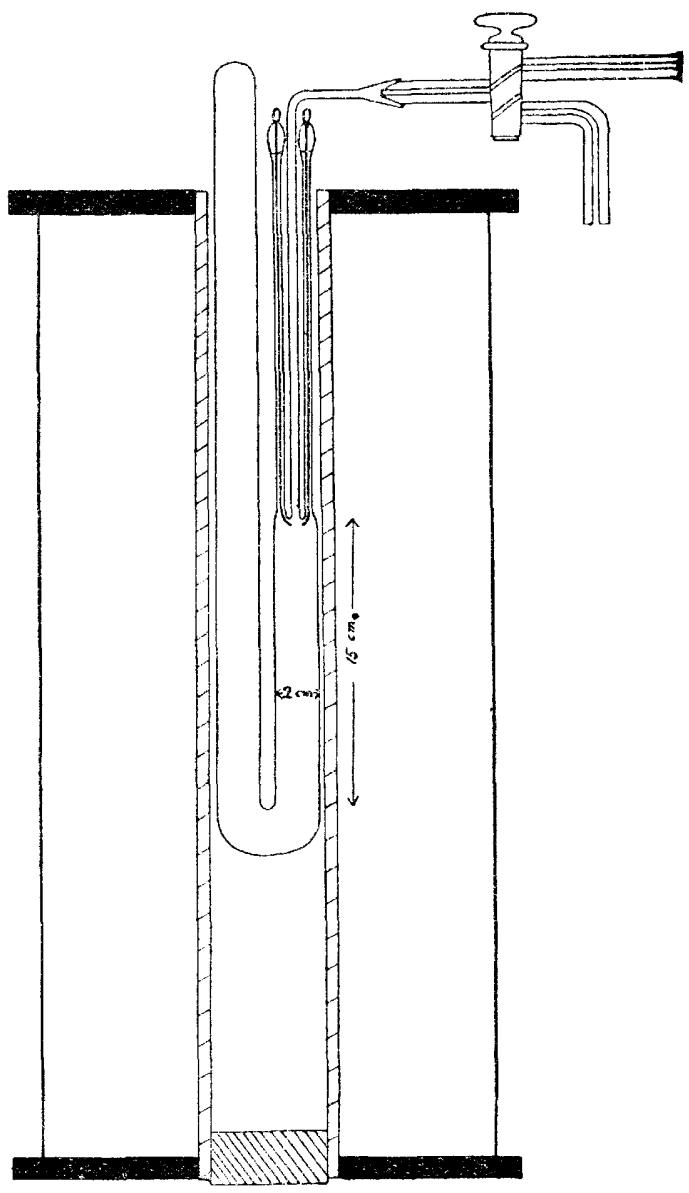

EXPER M E T A L.

The apparatus used for determining the limits of inflammability at different temperatures and at pressures less than atmospheric is shown in Fig. 3. It consisted essentially of a U-tube of transparent quartz, the limbs of which were close together, fixed vertically in a tube furnace which could be heated electrically. One limb of the U-tube was $15 \mathrm{~cm}$. long and had a capillary 
extension which was fitted, by means of a ground joint, with a glass three-way tap, of the form shown in the diagram, to make connexion with either a vacuum pump or a gasholder and mercury manometer. The other, longer, limb $(35 \mathrm{~cm}$.) was sealed at the top, which projected above the level of the furnace. The electrodes for igniting the mixtures were platinum wires led into the quartz tube, immediately below the capillary extension, through capillary side-tubes, the upper ends of which were closed by plugs of glass ground in, through which the platinum wires were sealed. The spark-gap was $4 \mathrm{~mm}$.

Since the limits for upward (or horizontal) propagation of flame are wider than for downward propagation, a mixture which on ignition enabled flame to travel just the full length of the shorter limb of the U-tube easily carried flame up the longer limb, and its appearance could be observed in the portion of the tube above the furnace, either directly or, as was found more convenient during experiments at low pressures, in a mirror inclined at an angle of $45^{\circ}$ fixed above the top of the tube and enclosed in a darkened box.

Ignition was caused by a short series of sparks from an " 8 -inch" $X$-ray coil, with a current of 15 amperes in the primary circuit. The intensity of this discharge with a $4 \mathrm{~mm}$. spark-gap was well in excess of the minimum required for the least easily ignitible mixtures, whilst the length of the tube was sufficient to ensure that the initial impetus imparted to the flames by the source of ignition had died away before the bottom was reached.

For the experiments at high pressures, a tube of thick glass $18 \mathrm{~cm}$. long and of $2 \mathrm{~cm}$. internal diameter was employed, fixed vertically. The tube was provided at the top with a high-pressure three-way tap of capillary bore, to make connexion with either a vacuum pump or a condensing syringe in communication with a gas-holder containing the mixture to be experimented with. A high-pressure tap at the bottom of the tube communicated with a Bourdon pressure gauge. The electrodes were platinum wires sealed into the glass at the top of the tube, the spark-gap being $4 \mathrm{~mm}$. A secondary discharge of the same intensity as for the experiments in the quartz tube was used to ignite the mixtures. The lower third of the tube was covered on the outside with black paper, in which a horizontal slit $2 \mathrm{~mm}$. wide was cut at a distance of $15 \mathrm{~cm}$. from the point of ignition; flame was judged to have travelled throughout the tube if it was observed to pass this slit.

For the experiments at different temperatures, the method of procedure was to obtain two mixtures, differing by 0.10 per cent. of methane, the one enabling flame to appear in the longer limb 
of the quartz U-tube, whilst with the other no flame appeared. The mean percentage of methane in the two mixtures was taken as the limiting percentage. For the experiments at different pressures, two pressures were obtained for a given mixture, differing by $10 \mathrm{~mm}$. of mercury at pressures below atmospheric or by $50 \mathrm{~mm}$. at higher pressures, such that at one pressure flame appeared in the prescribed manner, whilst at the other it did not. The mean pressure was taken as the limiting pressure for the particular mixture under the conditions of the experiment.

The methane was obtained from a natural source of firedamp. and purified by liquefaction. The mixtures with air (free from carbon dioxide) were prepared in glass gas-holders over glycerol and water, and were analysed before use.

[Received, November 23rd, 1917.] 